\title{
Electric and Magnetic Coulomb Potentials in the Deuteron
}

\author{
Bernard Schaeffer \\ 7, rue de 1'Ambroisie, 75012 Paris, France \\ E-mail: bschaeffer@wanadoo.fr
}

\begin{abstract}
After one century of nuclear physics, the underlying fundamental laws of nuclear physics are still missing. Bohr had found a formula for the $\mathrm{H}$ atom and another for the $\mathrm{H} 2$ molecule but no equivalent formula exists for the deuteron $2 \mathrm{H}$. The only known Coulomb interaction in a nucleus by the mainstream nuclear physics is the long range repulsion between protons, forgetting that the neutron contains electric charges with no net charge. The neutron is attracted by the proton in a way discovered two millenaries ago by the Greeks. This attraction is equilibrated by the repulsion between the opposite magnetic moments of the proton and of the neutron in the deuteron. The bare application of geometry together with electric and magnetic Coulomb's interactions accounts for the binding energy of the deuteron, without fitting, with only 4 per cent discrepancy, proving the electromagnetic nature of the nuclear energy.
\end{abstract}

\section{Introduction}

In 1924 Bieler, of the Rutherford laboratory where the atomic nucleus was discovered, wrote:

"as the angle increases, the ratio of the actual scattering to what would be expected on the inverse-square law diminishes rapidly. This suggests the existence of an attractive force at short distances from the nucleus". He made an unsuccessful "attempt to explain on a magnetic hypothesis this inverse fourth-power term in the law of force"[1].

The neutron was discovered in 1931 by his colleague, Chadwick. The neutron seeming to be uncharged, the electromagnetic hypothesis for the nuclear interaction was abandoned. The magnetic moments of the proton and of the deuteron were discovered in 1932 by Stern and the magnetic moment of the neutron in 1938 by Bloch. Assuming the additivity of the magnetic moments, he found that, in the deuteron, the magnetic moment of the neutron was opposite to that of the proton. The existence of a magnetic moment in the neutron proved the existence of electric charges and currents in the neutron indicating that it is not an elementary particle, as it carries no net charge but still interacts with a magnetic field [2].

The assumption that "it is possible to fit all the lowenergy results by the assumption that the nuclear force between two protons is the same as that between a neutron and a proton" [3] is wrong because the proton and the neutron have different electric charges and magnetic moments. Dozens of forces have been imagined but their fundamental laws and constants remain "terra incognita". In spite of the discovery of the neutron magnetic moment and electric charges with no net charge, electric and magnetic interactions between nucleons are generally ignored in nuclear physics. The Coulomb barrier only is sometimes taken into account in few-nucleon interactions $[4,5,6]$.

The principle of the attraction between a proton and a neutron can be found in the book by Feynman [7]: "The positive charge attracts negative charges to the side closer to itself and leaves positive charges on the surface of the far side. The attraction by the negative charges exceeds the repulsion from the positive charges, there is a net attraction". The proton attracts the neutron in the same way as amber (elektron) attracts light objects as was discovered by the Greeks. They also discovered the magnetic properties of magnetite from mount Magnetos. Coulomb found the the corresponding fundamental laws.

In the deuteron, the electrostatic attraction between the proton and the neutron is equilibrated by the repulsion between the opposite magnetic moments of the proton and the neutron. The magnetic interaction between the nucleons is attractive or repulsive depending on the position and orientation of their magnetic moments. First results have been obtained for hydrogen and helium isotopes [8, 9]. Static electric and magnetic Coulomb forces alone explain the binding energy of a nucleus without fitting as will be shown below for the deuteron. Different approximations are shown in this paper for the deuteron.

\section{Calculation of the electric and magnetic Coulomb energies}

\subsection{General formula of the electric and magnetic Coulomb's laws combined}

The electric and magnetic Coulomb potential energies between nucleons may be united in a single formula : $[10,11$, 12]:

$$
\begin{array}{r}
U_{e m}=\sum_{i} \sum_{i \neq j} \frac{q_{i} q_{j}}{4 \pi \epsilon_{0} r_{i j}} \\
+\sum_{i} \sum_{i \neq j} \frac{\mu_{0}}{4 \pi r_{i j}^{3}}\left[\vec{\mu}_{i} \bullet \vec{\mu}_{j}-\frac{3\left(\vec{\mu}_{i} \bullet \vec{r}_{i j}\right)\left(\vec{\mu}_{j} \bullet \vec{r}_{i j}\right)}{r_{i j}^{2}}\right]
\end{array}
$$

The first term is the sum of the electrostatic interaction energy potential between electric charges $q_{i}$ and $q_{j}$ separated by $r_{i j}$. The second term is the magnetic interaction energy potential between nucleons with magnetic moments $\overrightarrow{\mu_{i}}$ and $\overrightarrow{\mu_{j}}$, separated by $\overrightarrow{r_{i j}}$. 


\section{Deuteron electromagnetic structure}
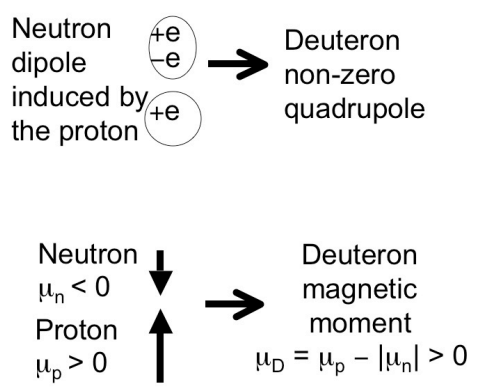

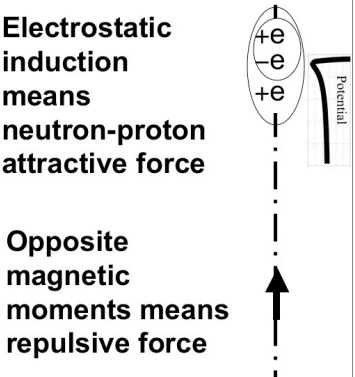

Figure 1: Schematic deuteron structure. - The elementary charges are assumed to be punctual. The proton contains an elementary charge $+e$. The neutron contains electric charges with no net charge, assumed to be $+e$ and $-e$. The electric charges of the neutron are separated by $2 a$ by the electric field of the proton distant by $r_{n p}$. The proton attracts electrically the neutron as a rubbed plastic pen attracts small pieces of paper. The magnetic moments of the proton and the neutron are opposite. They are also collinear in the deuteron by reason of symmetry. Their magnetic interaction equilibrates the electrostatic attraction.

\subsection{Energy of three collinear electric point charges}

The electric potential energy $U_{e}$ of this system of three point charges is, from formula (1):

$$
U_{e}=\frac{1}{4 \pi \epsilon_{0}}\left(\frac{q_{1} q_{2}}{r_{12}}+\frac{q_{2} q_{3}}{r_{23}}+\frac{q_{3} q_{1}}{r_{31}}\right)
$$

where $q_{1}, q_{2}$, and $q_{3}$ are the electric charges of the three charges, and $r_{12}, r_{23}$, and $r_{31}$ are their separation distances along their common axis. The deuteron nucleus is composed of one proton and one neutron. The proton contains a total electric charge $+e$. The neutron contains electric charges with no net charge. A simplifying assumption is to consider that it contains one $+e$ and one $-e$ electric charges.

\subsection{Deuteron electrostatic energy potential}

The permanent dipole of an isolated neutron is negligible but an induced electric dipole may be created by induction from a nearby proton whose electric charge $+e$ acts on the neutron electric charges with no net charge assumed to be $+e$ and $-e$ in a first approximation. $r_{n p}$ is the distance between the centers of the proton and the neutron. $2 a$ is the separation distance between the assumed punctual positive and negative charges of the neutron as shown on figure 1 . Applying formula (2) gives the total deuteron electric energy potential:

$$
U_{e}=\frac{e^{2}}{4 \pi \epsilon_{0}}\left(\frac{1}{r_{n p}+a}-\frac{1}{r_{n p}-a}-\frac{1}{2 a}\right)
$$

In the absence of the electric field, at large distances from a proton, the electric charges of the neutron are not separated,

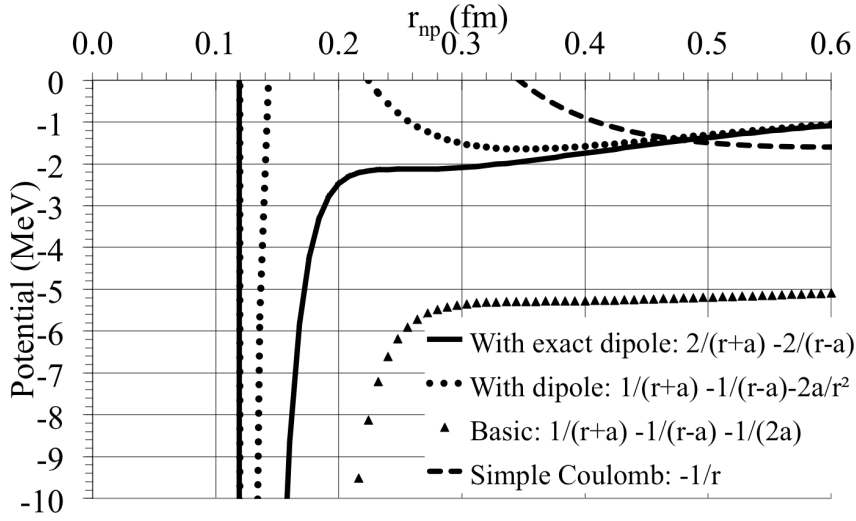

Figure 2: Calculated deuteron electromagnetic potential. - Four electrostatic approximations have been tested, the magnetic repulsion being standard. From top to bottom: exact dipole formula doubled, Coulomb plus dipole formula, basic Coulomb for three interacting charges and simple Coulomb neglecting the positive charge of the neutron. The exact formula gives the best result, $-2.13 \mathrm{MeV}$ (continuous line above) for $-2.225 \mathrm{MeV}$ experimental, thus a discrepancy of $4 \%$. This result is obtained by trial and error by varying the distance $2 a$ between the electric charges of the neutron. The equilibrium should be obtained at the minimum of the potential as was obtained by neglecting the positive charge of the neutron [8]. Unfortunately, the minimum is only a horizontal flat inflection point attributed to the Coulomb singularity at point charges.

probably concentric, $a=0$. For an isolated neutron, the formula diverges. The preceding formula (3) is thus not applicable here.

The usual approximate dipole formula, valid only when $a \ll r_{n p}$, doesn't work either when $a$ and $r_{n p}$ are of the same order of magnitude :

$$
U_{e}=\frac{e^{2}}{4 \pi \epsilon_{0}}\left(\frac{1}{r_{n p}+a}-\frac{1}{r_{n p}-a}-\frac{2 a}{r_{n p}^{2}}\right)
$$

It is thus necessary to apply the exact electric dipole formula [7], showing the attractive force between the positive and negative charges of the neutron:

$$
U_{\text {dipole }}=\frac{e^{2}}{4 \pi \epsilon_{0}}\left(\frac{1}{r_{n p}+a}-\frac{1}{r_{n p}-a}\right)
$$

The exact electric dipole formula (5) is exactly the same as the two first terms of the two preceding equations $(3,4)$. The total interaction energy between the proton and the neutron is thus twice the energy of an isolated dipole.

$$
U_{e}=\frac{e^{2}}{4 \pi \epsilon_{0}}\left(\frac{2}{r_{n p}+a}-\frac{2}{r_{n p}-a}\right)
$$

The total electric potential between the three electric charges $+e$ of the proton, $-e$ and $+e$ of the neutron is negative, thus attractive contradicting the usual assumption that 
the Coulomb force is zero in the deuteron (it is not yet possible to use the quarks whose physical properties are not well known).

\subsection{Deuteron magnetic energy potential}

In contrast with the electrostatic interaction, there is only one possibility for the magnetic interaction, the magnetic charges and monopoles being unlikely to be observed. According to formula (1), the magnetic potential energy of the deuteron is:

$$
U_{m}=\frac{\mu_{0}}{4 \pi r_{n p}^{3}}\left[\vec{\mu}_{n} \bullet \vec{\mu}_{p}-\frac{3\left(\vec{\mu}_{n} \bullet \vec{r}_{n p}\right)\left(\vec{\mu}_{p} \bullet \vec{r}_{n p}\right)}{r_{n p}^{2}}\right]
$$

The magnetic potential is positive, repulsive because the magnetic moments of the proton and the neutron in the deuteron are collinear and opposite $\left(\vec{\mu}_{n} \bullet \vec{\mu}_{p}<0\right)$ as shown on figure 1. The coefficient in the brackets is thus equal to $2\left|\mu_{n} \mu_{p}\right|$ :

$$
U_{m}=\frac{\mu_{0}}{4 \pi} \frac{2\left|\mu_{n} \mu_{p}\right|}{r_{n p}^{3}}
$$

\subsection{Deuteron Coulomb electric and magnetic energy potentials combined}

Adding the electrostatic (11) and magnetostatic (8) components of the Coulomb electromagnetic potential, formula (1) becomes :

$$
U_{e m}=\frac{e^{2}}{4 \pi \epsilon_{0}}\left(\frac{2}{r_{n p}+a}-\frac{2}{r_{n p}-a}\right)+\frac{\mu_{0}}{4 \pi}\left(\frac{2\left|\mu_{n} \mu_{p}\right|}{r_{n p}^{3}}\right)
$$

or, numerically:

$$
U_{e m}=1.442\left(\frac{2}{r_{n p}+a}-\frac{2}{r_{n p}-a}\right)+\frac{0.170}{r_{n p}^{3}} \mathrm{MeV}
$$

There is one variable $r_{n p}$ and one parameter $a$ in formula (10). In order to find the binding energy it is necessary to adjust the parameter a of the curve to obtain a potential minimum for both $r_{n p}$ and $a$. This is not to be confused with fitting to adjust the binding energy values as practiced in mainstream nuclear physics. Due to the Coulomb singularity, the potential has only a local minimum, a horizontal inflection point. The curve of the electromagnetic potential is shown on figure 2 , continuous dark line, calculated with formula (10). The horizontal inflection part of the curve corresponds to the deuteron binding energy. The result, obtained by applying electric and magnetic Coulomb's laws and the corresponding fundamental constants, is in accord with the experimental value of the deuteron binding energy $-2.225 \mathrm{MeV}$ for $-2.13 \mathrm{MeV}$ calculated (4\% weaker).

\section{Discussion}

In a first calculation, an absolute minimum of the potential had been obtained [9] by neglecting the positive charge of the neutron with a potential minimum error of $30 \%$ as can be seen on figure 2, curve called "Simple Coulomb", although remaining in the right order of magnitude. Although this formula is more complicated and less precise than the Bohr formula for the $H$ atom, it is an analytical formula useful to understand the physics of the binding energy in the nucleus.

Using the exact formula for the dipole, the curve called "With exact dipole", shows no real minimum, only a flat inflection point. A real minimum could be obtained by modifying Coulomb's law as Yukawa did, $-g^{2} e^{-\lambda r} / r$ [13] where the "Two constants $g$ and $\lambda$ appearing in the above equations should be determined by comparison with experiment". These constants are not fundamental and have never been declared as such: it is only an ad hoc fitting. The calculations shown in this paper are easily verifiable and cannot be coincidental because it is the strict application of Coulomb's electric and magnetic fundamental laws together with the experimentally known properties of the individual nucleons and of the deuteron structure. The electromagnetic approach explains quantitatively[14] why the binding energy per nucleon of the $\alpha$ particle $4 H e$ is six times larger than that of the deuteron ${ }^{2} H$ usually qualitatively explained because ${ }^{2} \mathrm{H}$ is loosely bound and ${ }^{4} \mathrm{He}$ tightly bound.

\section{Conclusion}

The binding energy of the deuteron has been calculated by applying the electric and magnetic Coulomb's laws, knowing that the deuteron contains the electric charge $+e$ of the proton, plus the neutron electric charges with no net charge, assumed to be $+e$ and $-e$. The magnetic moments of the proton and the neutron being opposite in the deuteron (figure 1), the electric attraction between the proton and the neutron is equilibrated by their magnetic repulsion, as the centrifugal force in the Bohr atom equilibrates the electric attraction. The graph (figure 2, continuous dark curve called "With exact dipole") shows the calculated nuclear potential of the deuteron where the horizontal inflection point matches with the binding energy of the deuteron with a $4 \%$ discrepancy only. Although less precise, similar results have been obtained for $H$ and $H e$ isotopes [14, 15]. Agreement between theory and experiment proves the electromagnetic nature of the nuclear interaction, contradicting the mainstream theory, based on a mysterious strong force whose fundamental laws and constants are unknown. Up to now, the huge amount of energy in the nuclear bonds, known to be around one million times the chemical bond energy had never been explained from fundamental laws. Only the electric and magnetic Coulomb's laws have been able to explain quantitatively the nuclear energy, opening a new application of electromagnetism. 


\section{Appendix : Fundamental Constants Used [16]}

- Light velocity:

$$
c=299272013 \mathrm{~m} / \mathrm{s}
$$

- Proton-electron mass ratio:

1836.152672

- Fine structure constant:

$$
\alpha=\frac{e^{2}}{2 \epsilon_{0} h c}=\frac{1}{137.035999679}
$$

- Proton mass:

$$
m_{p}=938.272013 \mathrm{MeV}
$$

- Proton Compton radius:

$$
R_{P}=\frac{\hbar}{m_{p} c}=0.21030890861 \mathrm{fm}
$$

- Nuclear magneton:

$$
\mu_{N}=\frac{e \hbar}{2 m_{p}}=\frac{e c R_{P}}{2} J . T^{-1}=5.05078324 J . T^{-1}
$$

- Magnetic moments of the neutron and the proton: $\mu_{n}$ and $\mu_{p}$ and their corresponding Landé factors,

$$
\mu_{i}=\frac{g_{i}}{2} \mu_{N}=g_{i} R_{P} \frac{e c}{4} J . T^{-1}
$$

where $i$ means $n$ or $p$.

- Relation between vacuum dielectric permittivity and magnetic permeability:

$$
\epsilon_{0} \mu_{0} c^{2}=1
$$

- Nuclear electrostatic energy constant:

$$
\frac{e^{2}}{4 \pi \epsilon_{0} R_{P}}=\frac{\alpha \hbar c}{R_{P}}=\alpha m_{p} c^{2}=6.84690165 \mathrm{MeV}
$$

This fundamental constant, $4 \%$ weaker than the $\alpha$ particle binding energy per nucleon $(-7.07 \mathrm{MeV})$, is the nuclear equivalent of the Hartree energy or twice the hydrogen atom binding energy (Rydberg constant).

- Nuclear magnetic energy constant:

$$
\frac{\mu_{0}\left|\mu_{n} \mu_{p}\right|}{4 \pi R_{P}^{3}}=\alpha m_{p} c^{2} \frac{\left|g_{n} g_{p}\right|}{16}=9.147871896 \mathrm{MeV}
$$

\section{References}

[1] E.S. Bieler, The large-angle scattering of $\alpha$-particles by light nuclei, Proc. R. Soc. Lond. A 105 (1924) 434450 .

[2] F. Bloch, Le moment magnétique du neutron, Annales de l'I.P.H.P. 8 (1938) 63-78 .

[3] V.F. Weisskopf, J.M. Blatt, Theoretical Nuclear Physics (Courier Dover Publications, 1991).

[4] St. Kistryn et al., Evidence of the Coulomb-force effects in the cross-sections of the deuteron-proton breakup at $130 \mathrm{MeV}$, Phys.Lett. B641 (2006) 23-27.

[5] A. Kievsky, M. Viviani, L. E. Marcucci, N-d scattering including electromagnetic forces, Phys. Rev. C 69, (2004) 014002.

[6] I. Hornyak, A.T. Kruppa, Two-body Coulomb scattering and complex scaling, Phys. Rev., A 85, 022702 (2012).

[7] R. Feynman, R. B. Leighton, M. Sands, The Feynman Lectures on Physics 2, Pearson/Addison-Wesley, Reading, Mass, 2006.

[8] B. Schaeffer, Electromagnetic theory of the nuclear interaction. Application to the deuteron ${ }^{2} H$, Frontiers of Fundamental Physics, 6-9 July 2010, Paris, France, AIP Conf. Proc. 1446, 123-131 (2012).

[9] B. Schaeffer, Electromagnetic Theory of the Binding Energy of the Hydrogen Isotopes, Journal of Fusion Energy, 30 (2011) 377-381.

[10] J.C. Maxwell, A treatise on electricity and magnetism, Vol.2, Oxford University Press, 1998.

[11] G.E. Owen, Introduction to Electromagnetic Theory, Courier Dover Publications, Oxford, 2003.

[12] K. Yosida, Theory of magnetism, Springer-Verlag, Berlin, 1996.

[13] H. Yukawa, On the Interaction of Elementary Particles, Proc. Phy. Math. Soc. Japan 17 (1935) 48-57

[14] B. Schaeffer, Ab Initio Calculation of ${ }^{2} \mathrm{H}$ and ${ }^{4} \mathrm{He}$ Binding Energies, Journal of Modern Physics, 3, 2012, 1709-1715.

[15] B. Schaeffer, Electromagnetic Nature of Nuclear Energy: Application to $\mathrm{H}$ and $\mathrm{He}$ Isotopes, World $\mathrm{J}$. Nucl. Sci. Techn., 3, 2013, 1-8.

[16] P. J. Mohr, B. N. Taylor, D. B. Newell, CODATA recommended values of the fundamental physical constants: 2010, Rev. Mod. Phys. 84, 1527-1605 (2012). 